INPLASY

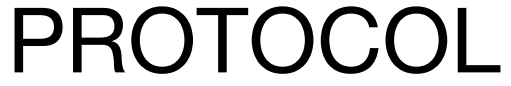

To cite: Zheng et al. Prognostic value of serum phosphate in critically ill patients: a metaanalysis. Inplasy protocol 2021120130. doi:

10.37766/inplasy2021.12.0130

Received: 28 December 2021

Published: 29 December 2021

Corresponding author:

Hui-Bin Huang

hhba02922@btch.edu.cn

Author Affiliation:

Beijing Tsinghua Chang Gung Hospital, Tsinghua University.

Support: None.

Review Stage at time of this submission: Data extraction.

Conflicts of interest:

None declared.

\section{Prognostic value of serum phosphate in critically ill patients: a meta-analysis}

Zheng, WH'1; Xu, Y2; Qin, JP3; Yao, Y4; Huang, HB5 .

Review question / Objective: We aim to perform a systemic review and meta-analysis to determine if abnormal serum phosphate (including Hypo- and Hyperphosphatemia) versus normal serum phosphate results in a statistically significant difference in outcomes in critically ill adult patients.

Condition being studied: Prognostic value of serum phosphate in critically ill patients. Authors of the current study come from a tertiary hospital in China and all the members have extensive experience in treating critical illness with CRRT. Furthermore, these authors have published several metaanalyses, which can guarantee the completion of the current study.

INPLASY registration number: This protocol was registered with the International Platform of Registered Systematic Review and Meta-Analysis Protocols (INPLASY) on 29 December 2021 and was last updated on 29 December 2021 (registration number INPLASY2021120130).

\section{INTRODUCTION}

Review question / Objective: We aim to perform a systemic review and metaanalysis to determine if abnormal serum phosphate (including Hypo- and Hyperphosphatemia) versus normal serum phosphate results in a statistically significant difference in outcomes in critically ill adult patients.

Condition being studied: Prognostic value of serum phosphate in critically ill patients. Authors of the current study come from a tertiary hospital in China and all the members have extensive experience in 
treating critical illness with CRRT. Furthermore, these authors have published several metaanalyses, which can guarantee the completion of the current study.

\section{METHODS}

Participant or population: Adult $(\geq 18$ years old) ICU patients.

Intervention: Abnormal serum phosphate (including Hypo- and Hyperphosphatemia).

Comparator: Normal serum phosphate.

Study designs to be included: No limitations.

Eligibility criteria: We will include studies focusing adults ( $\geq 18$ yr or older) critically ill patients with abnormal serum phosphate versus. normal serum phosphate.

Information sources: We will search the references in the included studies and personal files. We will request advice from experts in the field. Additionally, we will search associated articles from critical care, surgical, infection meetings; and contacted the authors of included trials, if need.

Main outcome(s): Primary outcome is longest follow-up mortality. Secondary outcomes include CRRT treatment requirement; mechanical ventilation free days; the length of stay in the ICU and hospital; $\triangle$ SOFA score and adverse events (as reported by authors).

Quality assessment / Risk of bias analysis: We will use Newcastle-Ottawa Scale to perform quality assessment/risk of bias analysis.

Strategy of data synthesis: An overall effect estimate for all data as risk ratio (RR) / mean difference (MD) with $95 \% \mathrm{Cl}$ will be calculated. The presence of statistical heterogeneity among the studies by using the $Q$ statistics and the heterogeneity by using the 12 statistic was addressed. A p value of less than 0.10 or an 12 value of greater than $50 \%$ as indicative was considered of substantial heterogeneity. A random-effects model or a fixed-effects mode (DerSimonian-Laird) will be chosen when significant eterogeneity or nonsignificant heterogeneity was not observed, respectively.

Subgroup analysis: We will perform subgroup analyses of all causes of mortality rates according to the patient gender, APPACHE II, Race; following up and patient source.

Sensitivity analysis: We will perform sensitivity analysis of all causes of mortality rates according to the period assessment (30-day, 90-day, ICU, or hospital mortality).

\section{Country(ies) involved: China.}

Keywords: Phosphate; meta-analysis; critically ill; mortality.

Contributions of each author:

Author 1 - When-He zheng.

Author 2 - Yuan Xu.

Email: xyuan76@163.com

Author 3 - Jun-Ping Qin.

Email: icuzhouhua@163.com

Author 4 - Yan Yao.

Author 5 - Hui-Bin Huang.

Email: hhba02922@btch.edu.cn 